\title{
Relação entre habilidades sociais, estresse, idade, sexo, escola e série em adolescentes
}

\author{
Sheila Francisca Machado, (iD) I, Sérgio Henrique de Souza Alves, (iD I Patrícia Fagundes Caetano, (D) II \\ ${ }^{I}$ Centro Universitário de Brasília, Brasília, DF, Brasil \\ II Universidade de Brasília, Brasília, DF, Brasil
}

\begin{abstract}
Resumo
Várias mudanças ocorrem no periodo da adolescência. O adolescente tem que desenvolver habilidades sociais, que são comportamentos que compõem o repertório do indivíduo para responder às demandas sociais adequadamente. Ele também enfrenta situações que podem desencadear estresse, que é a reação do organismo para reestabelecer seu equilíbrio após passar por situação estressora. O objetivo deste estudo foi verificar a relação de habilidades sociais e estresse com as variáveis: sexo, idade, tipo de escola e série. Foram utilizados os testes psicológicos ESA (Escala de Stress para Adolescentes) e IHSA-Del-Prette (Inventário de Habilidades Sociais para Adolescentes). Conforme os resultados encontrados no presente estudo, pode-se concluir que há correlação entre estresse e as variáveis sexo, idade e tipo de escola frequentada, assim como também entre a emissão de habilidades sociais e o tipo de escola frequentada. A hipótese de que as alunas teriam mais habilidades sociais que os alunos não foi confirmada, assim como também não se confirmou que os homens têm mais dificuldades na emissão das respostas do que as mulheres. Palavras-chave: habilidades sociais; estresse; adolescentes; gênero; tipo de escola.
\end{abstract}

\section{Relationship between social skills, stress, age, sex, school type and school grade in adolescents}

\begin{abstract}
During adolescence many changes occur in adolescent life. They have to develop social skills, behaviors that composes the repertoire of an individual to respond the social demands adequately. They face some situations that can cause stress, which is the reaction of the body to restore its balance after going through stressor situation. The present study aimed to investigate the correlation of social skills and stress variables: sex, age, type of school attended and grade. For this was used Psychological tests ESA (Stress Scale for Adolescents) and IHSA-Del-Prette(Inventory of Social Skills for Adolescents). The obtained results permit to conclude that there is a correlation between stress and the variables gender, age and type of school attended, as well as between the emission of social skills and the type of school attended. The hypothesis that the female students had more social skills than the students was not confirmed, nor it was confirmed that men have more difficulties in issuing the answers than women.
\end{abstract}

Keywords: social skills; stress; teenagers; gender; school type.

A adolescência é definida como período de transição entre a infância e a fase adulta. Começa por volta dos dez ou doze anos e vai até os dezoito e dezenove ou um pouco além. Nela ocorrem importantes mudanças físicas, cognitivas e psicossociais (PAPALIA; OLDS; FELDMAN, 2009). Não há consenso sobre o início da adolescência (PAPALIA; OLDS; FELDMAN, 2009; SALLES, 1998), que pode variar, dependendo da cultura e do autor. Pappalia, Olds e Feldman (2009) e Salles (1998) apontam o fato de a adolescência ser uma construção social, ligada às tradições culturais e a estereótipos divulgados pela mídia. O conceito aparece apenas no século XX; antes disso as crianças eram consideradas adultas a partir do momento em que aprendiam uma profissão ou amadureciam fisicamente.

Carvalho e Pinto (2002) comentam que o adolescente passa por mudanças corporais, maturação sexual, alteração de hormônios, que modificam seu corpo e impactam sua imagem pessoal. Ele adquire também novas capacidades cognitivas e responsabilidades relativas a papeis sociais que passa a desempenhar, já que tem a tarefa de

\footnotetext{
^Endereço para correspondência: Centro Universitário de Brasília, SEPN 707/907, Asa Norte, Brasília-DF. CEP 70790-075. E-mails: sheila machado@ hotmail.com, sergiohenrique.alves@gmail.com, patriciafag@gmail.com

Os dados completos dos autores encontram-se ao final do artigo.
}

construir sua identidade enquanto atende a expectativas e exigências de diversos grupos (família, amigos, comunidade em que vive), desenvolvendo gradativamente a capacidade de decisão, autonomia, discernimento e autorregulação do comportamento.

Carvalho e Pinto (2002) trazem também à reflexão a mudança da visão sobre os pais. O adolescente deixa de vê-los como heróis, buscando segurança nos grupos de amigos para recuperar a proteção que antes via na família. A inserção em grupos de amizade, contudo, também traz angústias, pois gera a necessidade de construção de novos valores e regras que auxiliem o adolescente a adaptar-se ao ambiente social. O apoio dos diversos meios sociais ajuda o desenvolvimento da autoestima, autoconfiança, responsabilidade e de outros atributos existentes nas relações interpessoais.

Vários autores comentam os fatores estressores do período da adolescência, tais como as transformações físicas e psicológicas, pressão com relação ao desempenho escolar e vestibular, intimidações por parte de colegas, gerando necessidade de ações em prol da autoafirmação, a escolha profissional, a entrada no mercado de trabalho. Assim, o adolescente passa por um período marcado pela ansiedade, medo, incertezas, construção da autoimagem 
e da autoestima, que podem desencadear estresse e depressão (PIRES, E.; PIRES, M.; PETROSKI, 2002; RIBEIRO, M.; RIBEIRO, L., 2005; ZIMPEL, 2005).

Del Prette, A. e Del Prette, Z. (2008) e Gresham (2009) chamam de habilidades sociais as diferentes classes de comportamentos que compõem o repertório social do sujeito para lidar com demandas das situações interpessoais adequadamente. As habilidades sociais podem ser consideradas como competências que ajudam na formação e manutenção de relacionamentos sociais positivos. Decodificar e selecionar, aperfeiçoar e decidir a melhor forma de agir em determinados contextos sociais são exemplos de habilidades aprendidas para atender às demandas sociais cotidianas.

Arón e Milicic (1994) comentam que o ambiente familiar é o primeiro a influir no desenvolvimento de habilidades sociais, já que o estilo familiar desenvolve modelos de comportamentos ao mesmo tempo em que molda condutas sociais por meio de práticas disciplinares, assim como ensina um sistema de crenças e valores que são transmitidos pelos componentes da família.

Segundo Del Prette, A. e Del Prette, Z. (2008), a adolescência é época de conquistas e descobertas em que o sujeito tem novas possibilidades cognitivas e o despertar da sexualidade, passando por instabilidade emocional devido às alterações hormonais que o corpo passa. Por isso, fazem-se necessárias ações educativas que estabeleçam regras e normas coniventes com valores morais da família.

Bandeira et al. (2006) investigaram a relação entre habilidades sociais e questões demográficas em alunos de $1^{\mathrm{a}}$ a $4^{\mathrm{a}}$ séries do ensino fundamental. Verificaram correlação negativa entre a idade e a autoavaliação das habilidades sociais. Nas meninas encontraram-se médias maiores do que nos meninos, havendo diferença significativa na avaliação das professoras, mas não na avaliação dos pais.

Com base em Arón e Milicic (1994), Del Prette, A. e Del Prette, Z. (2008) e Gresham (2009), tem-se uma lista de situações que ajudam a construir um repertório de habilidades sociais no adolescente: na família, na escola e com amigos. Tais fatores poderiam também ser fontes de estresse?

No campo da fisiologia, no século XX, Cannon (apud GONZÁLEZ, 2001) começou a falar em estresse como um estímulo, desenvolvendo daí a teoria da "reação de emergência" e destacando a função adaptativa das reações de medo e ira, expressas na liberação do hormônio adrenalina. Outro conceito importante descrito por Cannon (1939, apud LIPP, PEREIRA; SANDIR, 2005) é o de homeostase, a saber, a necessidade do organismo de manter constância de equilíbrio de forma automática para poder preservar sua existência.

O fisiologista Selye (1965) definiu estresse como reações sofridas pelo organismo ao entrar em contato com um evento com necessidade de esforço adaptativo. Assim, o estresse poderia ser entendido como forma de o corpo manter a homeostase. Lipp (2010) apresentou o conceito de stress como uma reação psicofisiológica frente a uma ameaça, com origem na necessidade do organismo de manter seu equilíbrio interno, diante de uma situação negativa ou desafiadora.

A situação que causa o estresse é chamada de estressor, definido como qualquer situação que quebre o equilíbrio interno, obrigando o sujeito a readaptar-se a fim de recobrar o equilíbrio. As situações podem ser vistas como estressantes pela sua natureza ou forma como são interpretadas (LIPP, 1996).

O estressor não é padronizado. Cada pessoa tem um estressor diferente de acordo com suas características pessoais, crenças, capacidade e comportamentos. Assim, não se pode generalizar quais eventos causarão estresse (OLIVEIRA, 1996). A resposta ao estresse depende da história de vida do sujeito, de suas características inatas, do apoio social e de outros componentes do sistema geral de relações interpessoais (GONZÁLEZ, 2001).

Durante o período da adolescência, várias situações estressoras podem ocorrer. Calais et al. (2007) encontraram em seu estudo com calouros e veteranos de jornalismo predominância de estresse entre mulheres.

Santos e Alves Júnior (2007), em pesquisa realizada com mestrandos da área de saúde, concluíram que mulheres são mais vulneráveis ao estresse, além de mostrar que os homens frequentemente focalizam mais os problemas como estratégia de enfrentamento do estresse. Os resultados encontrados pelos autores foram considerados por eles pouco fidedignos, devido à amostra ter sido composta por um número pequeno de participantes.

Na pesquisa de Machado, Veiga e Alves (2011), utilizando a Escala de Stress para Adolescentes (ESA), foi encontrada diferença significativa no fator interpessoal, comparando-se o resultado entre homens e mulheres. Adolescentes do sexo masculino obtiveram maiores escores.

Câmara e Carlotto (2007) investigaram a associação entre bem-estar psicológico e estratégias de enfrentamento do estresse em 389 estudantes do ensino médio. Observaram que meninas que assumiam como estratégia de enfrentamento uma postura ativa na resolução de problemas possuíam maior bem-estar psicológico, enquanto os meninos com maior bem-estar psicológico procuravam mais amigos íntimos, o sentimento de pertencimento e ajuda profissional.

Lipp et al. (2002) realizaram uma pesquisa com 255 alunos entre 7 e 14 anos. Verificaram diferença significativa nos níveis de estresse em relação ao tipo de escola que frequentavam (municipal, particular e particular confessional filantrópica). Um número maior de meninas apresentou estresse, e o nível deste diminuía com a elevação da série, sendo mais presente na primeira série.

Poucos estudos publicados investigaram estresse e habilidades sociais em adolescentes brasileiros, levando em conta variáveis como idade, série e tipo de escola frequentada pelo aluno. Assim, o presente estudo é importante por tentar identificar se há correlação das variáveis idade, sexo, tipo de escola e série com as variáveis habilidades sociais e estresse. 
Método

\section{Amostra}

Participaram da pesquisa 196 estudantes, metade da $2^{\mathrm{a}}$ série e metade da $3^{\mathrm{a}}$ série do ensino médio, com idade entre 15 e 18 anos, sendo 53,6\% do gênero feminino e $60,7 \%$ estudantes de escola particular.

\section{Instrumentos}

Foram utilizados dois testes psicológicos: 1. Escala de Stress Para Adolescentes - ESA, criada e validada por Tricoli (2005). Seu objetivo é verificar a presença de estresse em adolescentes na faixa etária de 14 a 18 anos. É constituída de 44 itens a serem avaliados em duas colunas, uma relativa ao período que o sintoma vem sendo sentido e a outra relativa à percepção do sintoma. A marcação é através de uma escala Likert de cinco pontos (1 a 5). O teste permite classificar o adolescente em "sem stress" ou "com stress" (TRICOLI, 2005). 2. Inventário de Habilidades Sociais para Adolescentes - IHSA-Del-Prete. Avalia habilidades sociais de adolescentes pelo autorrelato de situações comuns a essa faixa etária. Possui 38 itens que são avaliados em duas colunas, uma relativa à frequência com que o adolescente emite o comportamento e a outra, à dificuldade que ele tem em desempenhá-lo. O autorrelato é feito pela marcação de uma escala Likert de cinco pontos ( 0 a 4$)$. A soma dos itens de cada coluna possibilita classificar os adolescentes numa escala de percentil (DEL PRETTE, A.; DEL PRETTE, Z., 2009).

\section{Procedimento}

Pediu-se à escola autorização para a realização da pesquisa. Dada a autorização, o projeto foi submetido ao Comitê de Ética em Pesquisa. Ao entrar-se em sala de aula, explicou-se aos alunos a atividade para a qual estavam sendo convidados e foi-lhes entregue o Termo de Consentimento Livre e Esclarecido. Os testes foram aplicados após a entrega dos termos lidos e assinados. Os alunos foram orientados a preencherem no cabeçalho somente os campos sexo, idade, tipo de escola e série. Depois, foram lidas com eles as instruções dos testes. $\mathrm{O}$ tempo médio que levaram para responder a ambos os testes foi de 40 minutos.

\section{Análise dos dados}

Os dados foram analisados com a utilização do software estatístico Statistical Package for the Social Sciences versão 17. Foram realizadas análises de frequência e distribuição, objetivando verificar a existência de erro de digitação ou omissão de dados. Análises descritivas (média e desvio padrão) foram realizadas. Para comparação entre variáveis utilizou-se da correlação de Pearson, e o teste t no caso da análise de diferença entre gêneros.

\section{Resultados}

A Figura 1 apresenta a porcetagem de alunos do gênero feminino e do masculino com estresse. É possível observar que, dos 50 alunos que apresentaram estresse durante a apuração dos resultados do teste, $52 \%$ são do gênero masculino e $48 \%$ do feminino.
Figura 1: Porcetagem de alunos de cada gênero com estresse.

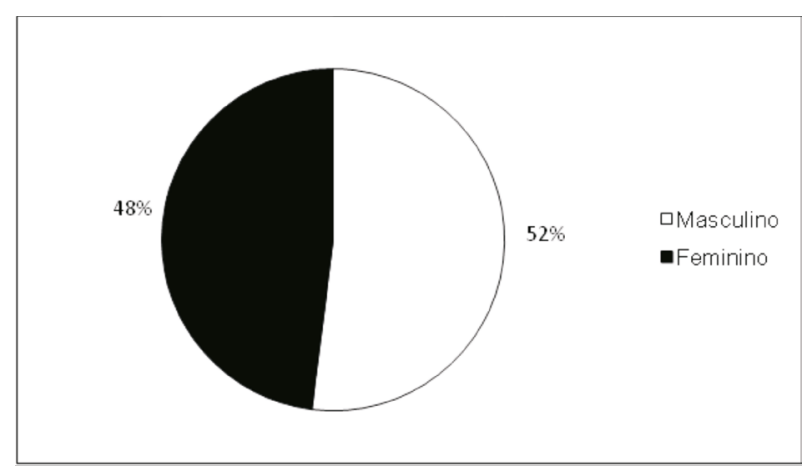

Na Figura 2 são apresentadas as distribuições das classificações do teste IHSA-Del-Prette apenas entre as participantes. Das 105 alunas que responderam ao teste, $42,86 \%$ ficaram abaixo da média inferior, $7,62 \%$ se encontram na média inferior, $20 \%$ apresentaram um bom repertório comportamental, 9,52\% um repertório comportamental elaborado e $20 \%$ um repertório comportamental altamente elaborado. Assim, 49,52\% das estudantes relatam ter habilidades sociais para atuar adequadamente em diversas situações, indicando recursos interpessoais satisfatórios; porém, $50,48 \%$ das alunas apresentaram indicativo de necessidade de treinamento de habilidades sociais.

Figura 2: Porcentagem de alunas em cada classificação de frequência do comportamento do teste IHSA-Del-Prette.

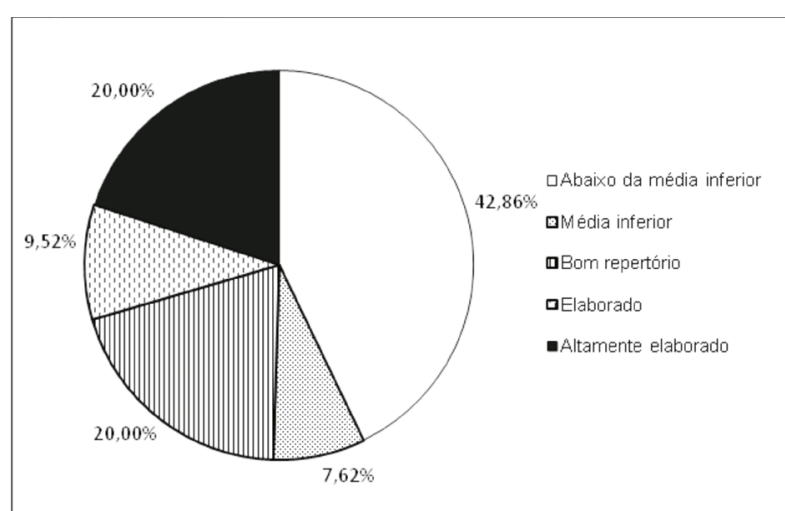

Na Figura 3 são apresentadas as classificações das participantes com relação ao fator dificuldade. É possível observar que $47,62 \%$ das alunas relatam baixo custo na emissão da resposta, enquanto outras 30,48\% relataram médio custo e $21,90 \%$, alto custo. Esses resultados sinalizam que a maioria não tem muitas dificuldades na emissão do comportamento, mesmo que tenham ansiedade na hora de se comportar.

Figura 3: Porcentagem de alunas em cada classificação de dificuldade do comportamento do teste IHSA-Del-Prette.

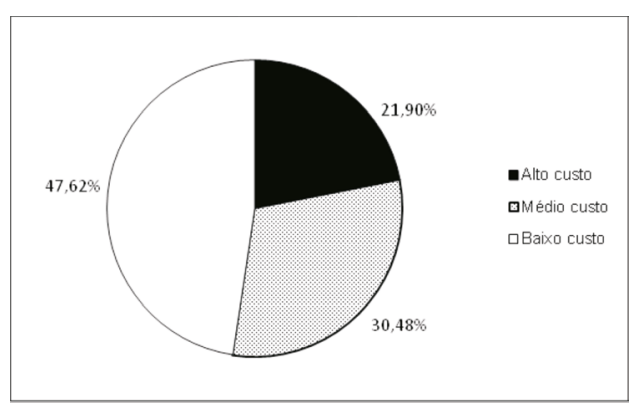

Fractal, Rev. Psicol., v. 32 - n. esp., p. 210-217, 2020 
Na Figura 4 são apresentadas as distribuições entre as classificações do teste IHSA-Del-Prette apenas entre os participantes. Encontram-se abaixo da média inferior $30,77 \%$ dos alunos, $15,38 \%$ se encontram na média inferior, 36,26\% apresentaram um bom repertório, 5,50\% um repertório elaborado e $12,09 \%$, um repertório altamente elaborado. Desse modo, $53,85 \%$ dos estudantes relatam ter habilidades sociais para atuar adequadamente em diversas situações, indicando recursos interpessoais satisfatórios. Contudo, 46,15\% dos alunos demonstraram indicativo de necessidade de treinamento em habilidades sociais.

Figura 4: Porcentagem de alunos em cada classificação de frequência do comportamento do teste IHSA-Del-Prette.

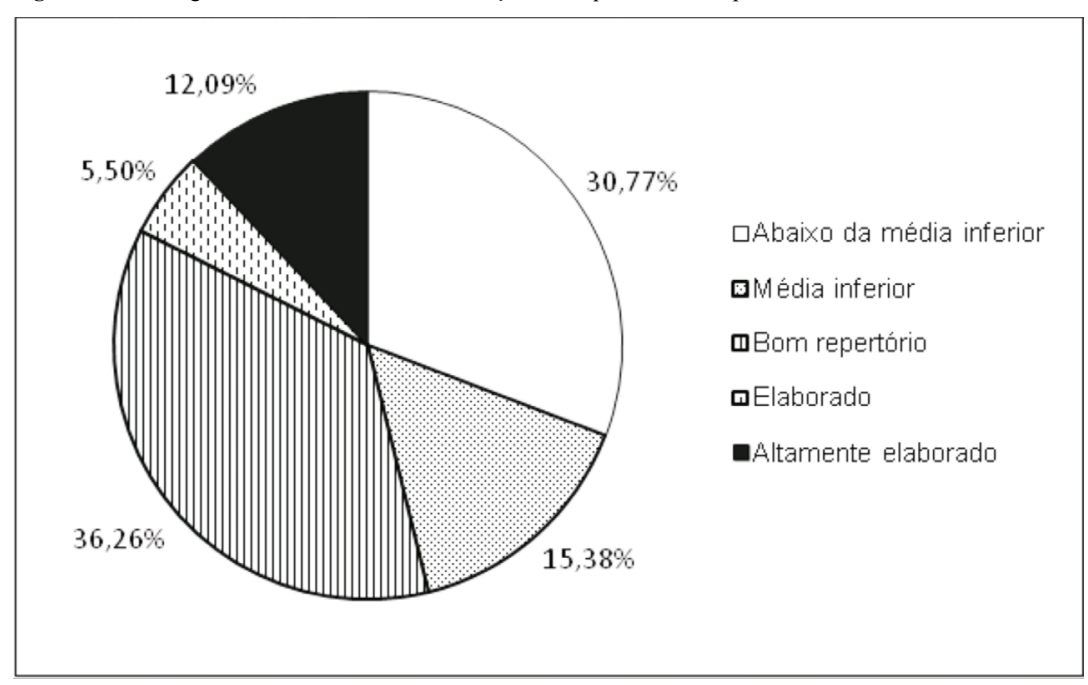

Na Figura 5 são apresentadas as classificações dos participantes com relação ao fator dificuldade. Vê-se que $50,55 \%$ relatam baixo custo na emissão dos comportamentos. Outros $32,97 \%$ relataram médio custo na emissão da resposta e $16,48 \%$, alto custo, sinalizando que a maioria não tem dificuldades na emissão do comportamento, mesmo que tenham ansiedade na hora de se comportar.

Abaixo da média inferior

Bom repertóri e $34,37 \%$, respectivamente). Houve mais alunos do gênero masculino $(61,11 \%)$ com "bom repertório" do que alunas $(38,89 \%)$. As alunas também representam a maior parte de alunos com percentis abaixo da média $(61,65 \%)$, enquanto que os alunos são maioria nos percentis que se encontram na média inferior $(63,63 \%)$.

A fim de se verificar a relação existente entre os testes ESA e IHSA-Del-Prette e a variável sexo, foi realizada análise de correlação de Pearson. Os resultados são mostrados na Tabela 2.
Figura 5: Porcentagem de alunos em cada classificação de dificuldade do comportamento do teste IHSA-Del-Prette.

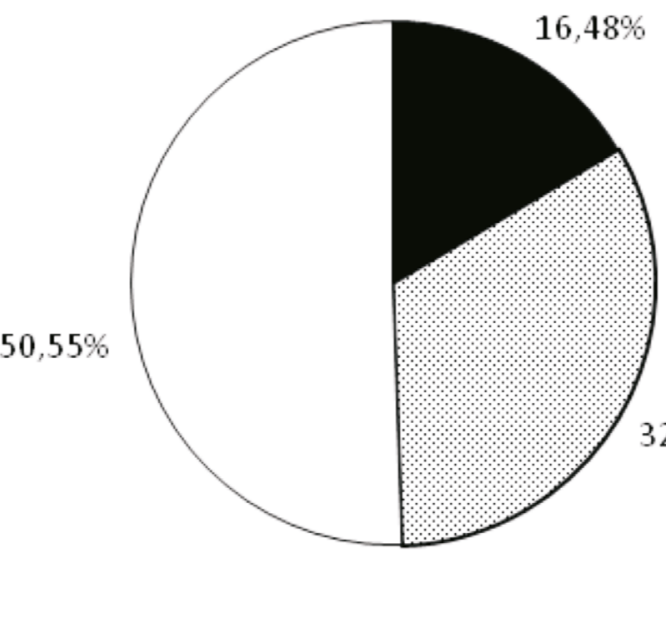

Alto custo

๑Médio custo

口Baixo custo
Nota-se que a única correlação foi entre a variável gênero e o fator Sintomas do teste ESA $(r=0,29$ $p<0,01)$. O fator Período do ESA e os dois fatores do IHSA-Del-Prette não apresentaram correlação com a variável sexo.

Na Tabela 3, são apresentadas as médias e desvios padrões dos testes ESA e IHSA-Del-Prette em relação à variável sexo. É possível observar que as mulheres apresentaram médias relativamente maiores em relação aos homens em todos os fatores.

A Tabela 4 mostra o resultado do teste t para amostras independentes. Os resultados mostram que há diferença significativa entre grupos nas variáveis no escore do fator Sintomas $(\mathrm{ESA}), t(194)=4,31(p<0,01)$.

Ao se considerar a correlação apresentada entre o fator Sintomas do ESA e a variável gênero $(r=0,29)$ e médias apresentadas por homens e mulheres nesse fator, verifica-se que as mulheres apresentam maiores médias do que os homens, ou seja, mais sintomas de estresse e por um período maior do que os homens, além de um repertório bem elaborado, mas com mais dificuldade ou ansiedade na apresentação dos comportamentos que os homens.

A Tabela 5 mostra que houve correlação da idade (sintoma $r=-0,18$ e período $r=-0,17 p>0,05)$ e do tipo de escola (sintoma $r=0,21$ e período $r=0,20 p>0,01$ )

A Tabela 1 mostra a porcentagem de alunos de cada gênero nas classificações do IHSA-Del-Prette. Vê-se que houve mais alunas na classificação "elaborado" $(66,67 \%)$ e "altamente elaborado" $(65,63)$ do que alunos $(33,33 \%$ com os escores gerais do ESA e do tipo de escola e o escores geral da frequência $(r=0,26 p>0,01)$ do IHSA-DEL-PRETTE. 
Tabela 1: Porcentagem de alunos de cada sexo na classificação do IHSA-Del-Prette.

\begin{tabular}{llllll}
\hline & $\begin{array}{l}\text { Abaixo } \\
\text { da média }\end{array}$ & $\begin{array}{l}\text { Média } \\
\text { inferior }\end{array}$ & $\begin{array}{l}\text { Bom } \\
\text { repertório }\end{array}$ & Elaborado & $\begin{array}{l}\text { Altamente } \\
\text { elaborado }\end{array}$ \\
\hline Masculino & $38,35 \%$ & $63,63 \%$ & $61,11 \%$ & $33,33 \%$ & $34,37 \%$ \\
Feminino & $61,65 \%$ & $36,37 \%$ & $38,89 \%$ & $66,67 \%$ & $65,63 \%$ \\
Total & $100 \%$ & $100 \%$ & $100 \%$ & $100 \%$ & $100 \%$ \\
\hline
\end{tabular}

Tabela 2: Correlação entre fatores gerais e gêneros.

\begin{tabular}{llllll}
\hline & & Sintomas & Período & Frequência & Dificuldade \\
\hline Sexo dos & Correlação de Pearson & 0,29 & 0,13 & 0,03 & 0,03 \\
respondentes & Sig. (2-tailed) & 0,00 & 0,06 & 0,76 & 0,66 \\
& $\mathrm{~N}$ & 196 & 196 & 196 & 196 \\
\hline
\end{tabular}

**Correlação é altamente significativa $<0.01$ e *Correlação é significativa $<0.05$

Tabela 3: Análises descritivas por gênero.

\begin{tabular}{llllll}
\hline & & Sintomas & Período & Dificuldade & Frequência \\
\hline Masculino & Média & 2,11 & 2,34 & 42,84 & 24,44 \\
& Desvio- & 0,53 & 0,79 & 20,26 & \\
& Padrão & & & 44,13 & 21,74 \\
Feminino & Média & 2,47 & 2,54 & 21,60 & 29,25 \\
& Desvio- & 0,61 & 0,73 & & \\
\hline
\end{tabular}

Tabela 4: Médias gerais por gênero em cada fator dos dois testes.

Teste para amostras independentes

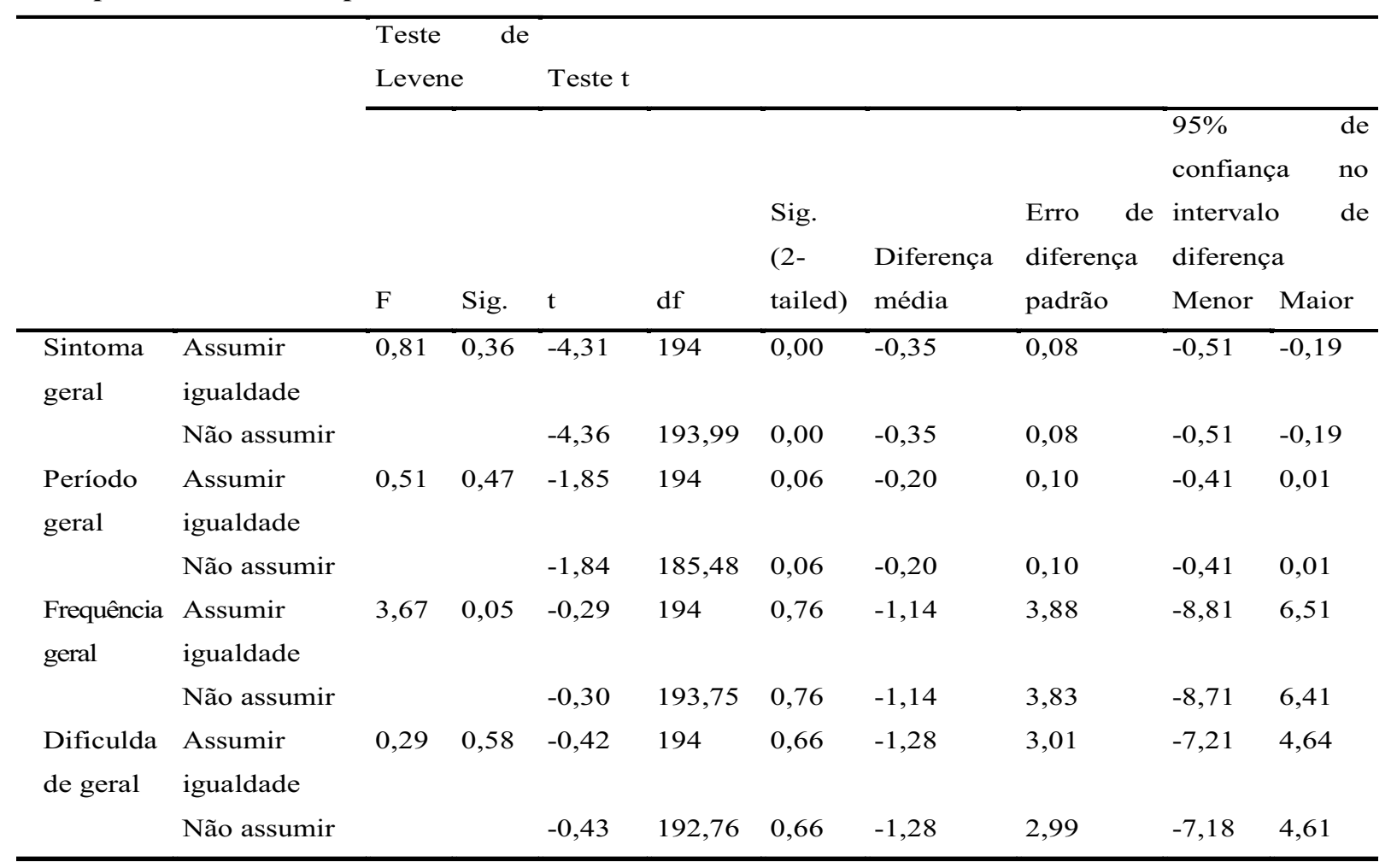


Tabela 5: Correlações entre os fatores dos testes e a idade, o tipo de escola e a série dos respondentes.

\begin{tabular}{|c|c|c|c|c|c|}
\hline & & $\overline{\text { Sintoma }}$ & $\overline{\text { Período }}$ & Frequência & $\overline{\text { Dificuldade }}$ \\
\hline Idade dos & Correlação de Pearson & $-0,18^{7}$ & $0,17^{*}$ & 0,01 & $-0,03$ \\
\hline \multirow[t]{2}{*}{ respondentes } & Sig. (2-tailed) & 0,01 & 0,012 & 0,88 & 0,63 \\
\hline & $\mathrm{N}$ & 196 & 196 & 196 & 196 \\
\hline \multirow[t]{3}{*}{ Tipo de escola } & Correlação de Pearson & $0 \longdiv { 2 1 * }$ & $\longdiv { 0 , 2 0 }$ & $0,2 \sqrt{6}$ & $-0,02$ \\
\hline & Sig. (2-tailed) & 0,003 & 0,004 & 0,00 & 0,70 \\
\hline & $\mathrm{N}$ & 196 & 196 & 196 & 196 \\
\hline Série que o & Correlação de Pearson & $-0,05$ & $-0,04$ & 0,05 & $-0,01$ \\
\hline está cursando & Sig. (2-tailed) & 0,44 & 0,56 & 0,41 & 0,81 \\
\hline respondente & $\mathrm{N}$ & 196 & 196 & 196 & 196 \\
\hline
\end{tabular}

\section{Discussão}

Em termos de diferença entre os sexos, os resultados apontam para uma correlação entre sexo e o fator Sintomas do ESA $(r=0,29 p<0,01)$. Nesse fator, as mulheres apresentaram média maior que os homens. Apenas no fator Sintomas a diferença chega a ser significativa, de acordo com a Tabela $4(t(194)=4,31 p<0,01)$. Calais et al. (2007) e Santos e Alves Júnior (2007) apontam as mulheres como mais vulneráveis aos sintomas de estresse, justificando essa diferença pelo acúmulo de atividades exercidas por elas. Porém, ao contrário das pesquisas de Lipp et al. (2002), Calais et al. (2007), Santos e Alves Júnior (2007) e Machado, Veiga e Alves (2011), no presente estudo, houve maior porcentagem de alunos com estresse do que alunas. Câmara e Carlotto (2007) puderam observar a forma com que cada um desses dois grupos lidava com o estresse e encontrou nas mulheres a busca pela resolução do problema, enquanto nos homens, a busca pelas interações interpessoais.

A diferença entre gêneros também aparece nas habilidades sociais, já que, por mais que grande parte da amostra feminina e masculina não tenha apresentado alto custo na emissão da resposta, uma porcentagem maior de alunas do que de alunos apresentou indicação de necessidade de treinamento de habilidades sociais (Figuras 2, 3, 4, 5). Porém, essa diferença não foi significativa. Bandeira et al. (2006) encontraram estudantes do sexo feminino com pontuação de habilidades sociais mais alta do que os do sexo masculino. Bartholomeu, Nunes, e Machado (2008) identificaram diferenças entre os sexos nos diferentes fatores do teste, além da questão da pontuação geral.

Com relação à idade, série e tipo de escola, apenas a idade (sintoma $r=-0,18$ e período $r=-0,17 p>0,05$ ) e, principalmente, o tipo de escola (frequência $r=0,26$, sintoma $r=0,21$ e período $r=0,20 p>0,01)$ apresentaram correlação considerável com algum fator de um dos testes, já que apenas o tipo de escola correlacionou-se com algum fator de ambos os testes. A série dos participantes não apresentou correlação em nenhum teste (Tabela 5). Bandeira et al. (2006) haviam encontrado correlação entre habilidades sociais e idade em alunos de $1^{\mathrm{a}}$ a $4^{\mathrm{a}}$ série do ensino fundamental; Polo, Hernández e Poza (1996), entre idade e estresse em escolares e universitários; Lipp et al. (2002), entre o tipo de escola e estresse.
Com base nos resultados encontrados no presente estudo, pode-se concluir que há correlação entre estresse e as variáveis sexo, idade e tipo de escola frequentada, assim como também entre a emissão de habilidades sociais e o tipo de escola frequentada.

A hipótese de que as alunas teriam mais habilidades sociais que os alunos não foi confirmada, assim como também não se confirmou que os homens têm mais dificuldades na emissão das respostas do que as mulheres. Os escores de ambos os sexos no teste de habilidades sociais foi muito próximo, não havendo, pois, diferença significativa (Tabelas 1 e 2).

Estudos qualitativos com adolescentes são uma possibilidade de pesquisa futura para compreender como esses fenômenos acontecem; outra seria um estudo com adolescentes de vários estados, permitindo comparações entre localidades; e uma terceira vertente poderia, ainda, comparar alunos de ensino médio com universitários.

\section{Informações sobre os autores:}

\section{Sheila Francisca Machado \\ (iD) https://orcid.org/0000-0002-0399-8772 \\ (9) http://lattes.cnpq.br/2503900250848581}

Possui graduação em Psicologia pelo Centro Universitário de Brasília (UniCEUB). Realizou pesquisas na área de avaliação psicológica. Colaborou em pesquisa sobre comportamento organizacional e realizou sua monografia na área da saúde do trabalho, realizando uma pesquisa de campo utilizando os conceitos da ergonomia da atividade. No Instituto Brasiliense de Análise do Comportamento (IBAC) concluiu o curso de Especialização em Análise comportamental Clínica. Concluiu também Especialização em Avaliação Psicológica no Instituto de Pós-Graduação de Goiás (IPOG).

\section{Sérgio Henrique de Souza Alves \\ (iD) https://orcid.org/0000-0001-8081-9267 \\ (9) http://lattes.cnpq.br/8265053024160718}

Possui graduação, mestrado e doutorado em Psicologia pela Universidade de Brasília. Atualmente é professor titular do Instituto de Educação Superior de Brasília e professor titular do Centro Universitário de Brasília. Realiza estudos voltados para a Psicobiologia, desenvolvendo principalmente os seguintes temas: labirinto em cruz elevado, ansiedade, anxiety, hippocampus e serotonina. Atua na área clínica como analista do comportamento. 
Patrícia Fagundes Caetano

(iD) https://orcid.org/0000-0003-1551-877X

(9) http://lattes.cnpq.br/5909810326144558

Atua desde 2003 como consultora na área de Gestão de Pessoas, principalmente em Avaliação Psicológica para Concursos Públicos, Análise Profissiográfica (estudo científico do cargo) e Perfil Psicológico. Possui experiência profissional, desde 2008, na área de Mapeamento de Competências, Perfil de Competências e Seleção por Competência para instituições públicas como Ministério da Justiça via Programa das Nações Unidas para o Desenvolvimento, PNUD, Secretaria de Defesa Social do Estado Minas Gerais, Secretaria de Segurança Pública do Estado de Tocantins, Polícia Civil do Distrito Federal, Secretaria do Orçamento do Ministério do Planejamento SOF/MP, Polícia Federal, Polícia Rodoviária Federal. Elaborou e validou um instrumento para avaliação de competências, intitulado: "Inventário de Competências Pessoais para o Trabalho" (ICPT). Em 2012, publicou como coautora na revista Psicologia: Ciência e Profissão o artigo: "Análise Profissiográfica e Mapeamento de Competências nas Instituições de Segurança Pública". Trabalha também como professora de disciplinas como Gestão de Pessoas, Análise Profissiogáfica, Gestão e Mapeamento de Competências, Avaliação Psicológica e Testes Psicológicos.

\section{Contribuições dos autores:}

Todos os autores colaboraram ao longo do processo, desde a elaboração até a revisão final do manuscrito. Os autores aprovaram o manuscrito final para publicação.

\section{Como citar este artigo:}

\section{ABNT}

MACHADO, Sheila Francisca; ALVES, Sérgio Henrique de Souza; CAETANO, Patrícia Fagundes. Relação entre habilidades sociais, estresse, idade, sexo, escola e série em adolescentes. Fractal: Revista de Psicologia, Niterói, v. 32, n. esp., p. 210-217, jun. 2020. https://doi.org/10.22409/1984-0292/v32_i-esp/39792

\section{APA}

Machado, S. F., Alves, S. H. S., \& Caetano, P. F. (2020, Junho) Relação entre habilidades sociais, estresse, idade, sexo, escola e série em adolescentes. Fractal: Revista de Psicologia, 32(esp.), 210-217. doi: https://doi.org/10.22409/1984-0292/v32_i-esp/39792

\section{Copyright:}

Copyright (C) 2020 Machado, S. F., Alves, S. H. S., \& Caetano, P. F. Este é um artigo em acesso aberto distribuído nos termos da Licença Creative Commons Atribuição que permite o uso irrestrito, a distribuição e reprodução em qualquer meio desde que o artigo original seja devidamente citado.

Copyright (C) 2020 Machado, S. F., Alves, S. H. S., \& Caetano, P. F. This is an Open Access article distributed under the terms of the Creative Commons Attribution License, which permits unrestricted use, distribution, and reproduction in any medium, provided the original article is properly cited.

\section{Referências}

ARÓN, Ana Maria; MILICIC, Neva. Viver com os outros: programa de desenvolvimento de habilidades sociais. Tradução de Jonas Pereira dos Santos. Campinas: Psy, 1994.

BANDEIRA, Marina et al. Competência acadêmica de crianças do ensino fundamental: características sociodemográficas e relação com habilidades sociais. Interação em Psicologia, v. 10, n. 1, p. 53-62, 2006. http://dx.doi.org/10.5380/psi.v10i1.5773
BARTHOLOMEU, Daniel; NUNES, Carlos Henrique Sancineto da Silva; MACHADO, Afonso Antonio. Traços de personalidade e habilidades sociais em universitários. PsicoUSF (Impr.), Itatiba, v. 13, n. 1, p. 41-50, 2008 https://doi. org/10.1590/S1413-82712008000100006

CALAIS, Sandra Leal et al. Stress entre calouros e veteranos de jornalismo. Estudos de Psicologia, Campinas, v. 24, n. 1, p. 6977, 2007. https://doi.org/10.1590/S0103-166X2007000100008

CÂMARA, Sheila Gonçalves; CARLOTTO, Mary Sandra. Coping e gênero em adolescentes. Psicologia em estudo, Maringá, v. 12, n. 1, p. 87-93, 2007. https://doi.org/10.1590/ S1413-73722007000100011

CARVALHO, Alysson; PINTO, Mario V. Ser ou não ser... Quem são os adolescentes? Navegar é preciso... In: CARVALHO, A.; SALlES, F.; GUIMARÃES, M. (Org.). Adolescência. Belo Horizonte: UFMG, 2002. p. 11-30.

DEL PRETTE, Almir; DEL PRETTE, Zilda Aparecida. Psicologia das relações interpessoais: vivências para o trabalho em grupo. Petrópolis: Vozes, 2008.

DEL PRETTE, Almir.; DEL PRETTE, Zilda Aparecida. Inventário de habilidades sociais para adolescentes: manual de aplicação, apuração e interpretação. São Paulo: Casa do Psicólogo, 2009.

GONZÁLEZ, Miguel Alvarez. Stress: temas de psiconeuroendocrinologia. Tradução de Maria Cristina T. V. Teixeira. 2. ed. São Paulo: Robe, 2001.

GRESHAM, Frank. Análise do comportamento aplicada às habilidades sociais. In: DEL PRETTE, Almir; DEL PRETTE, Zilda Aparecida (Org.). Psicologia das habilidades sociais: diversidade e suas implicações. Petrópolis: Vozes, 2009. p. 1766.

LIPP, Marilda Emmanuel Novaes (Org.). Stress: conceitos básicos. In: _. Pesquisas sobre stress no Brasil: saúde ocupações e grupos de risco. Campinas: Papirus, 1996. p. 1731.

LIPP, Marilda Emmanuel Novaes (Org.). O modelo quadrifásico do stress. In: Mecanismos neuropsicofisiológicos do stress: teoria e aplicações clínicas. São Paulo: Casa do Psicólogo, 2010. p. 17-22.

LIPP, Marida Emmanuel Novaes et al. O estresse em escolares. Psicologia escolar e educacional, Campinas, v. 6, n. 1, p. 51-56, 2002. http://dx.doi.org/10.1590/S1413-85572002000100006

LIPP, Marilda Emmanuel Novaes; PEREIRA, Márcia Bignotto; SADIR, Maria Angélica. Crenças irracionais como fontes internas de stress emocional. Revista Brasileira de Terapias Cognitivas, v. 1, n. 1, p. 29-34, 2005. Disponível em: $\quad$ http://pepsic.bvsalud.org/scielo.php?script=sci arttext\&pid=S1808-56872005000100004. Acesso em: 20 fev. 2019.

MACHADO, Sheila Francisca; VEIGA, Heila Magali da Silva; ALVES, Sérgio Henrique de Souza. Níveis de estresse em alunos de $3^{\text {a }}$ série do ensino médio. Universitas: Ciências da Saúde, Brasília, v. 9, n. 2, p. 35-52, 2011. http://dx.doi. org/10.5102/ucs.v9i2.1362

OLIVEIRA, Maria Amélia Vallim de. Administrando o stress com técnicas de programação neuroliguísticas. São Paulo: Gente, 1996. 
PAPALIA, Dianne; OLDS, Sally Wendkos; FELDMAN, Ruth Duskin. Desenvolvimento humano. São Paulo: McGraw-Hill, 2009.

PIRES, Edna Aparecida Goulart; PIRES, Mário César; PETROSKI, Edio Luiz. Adiposidade corporal, padrão de comportamento e estresse em adolescentes. Revista Brasileira de Cineantropometria e Desempenho Humano, Florianópolis, v. 4, n. 1, p. 7-16, 2002.

POLO, Antonia; LOPEZ, José Manuel Hernández; POZA, Carmen. Evaluación del estrés académico en estudiantes universitarios. Ansiedad y Estrés, Espanha, v. 2, n. 2-3, p. 159-172, 1996. Disponível em: https://pt.scribd.com/ document/39788022/Estres-Academicos-en-EstudiantesUniversitarios. Acesso em: 12 ago. 2019.

RIBEIRO, Marco Aurélio de Patrício; RIBEIRO, Luis Távora Furtado. Estresse: conhecer para superar. Petrópolis, RJ: Vozes, 2005.

SALLES, Leila Maria Ferreira. Adolescência, escola $e$ cotidiano: contradições entre o genérico e o particular. Piracicaba: UNIMEP, 1998.

SANTOS, André Faro; ALVES JÚNIOR, Antônio. Estresse e estratégias de enfrentamento em mestrandos de ciências da saúde. Psicologia: Reflexão e Crítica, Porto Alegre, v. 20, n. 1, p. 104-113, 2007. https://doi.org/10.1590/S010279722007000100014

SELYE, Hans. Stress: a tensão da vida. Tradução de Frederico Branco. São Paulo: IBRASA, 1965.

TRICOLI, Valquíria. Escala de stress para adolescentes. São Paulo: Casa do Psicólogo, 2005.

ZIMPEL, Rogério. Aprendendo a lidar com o estresse. São Leopoldo: Sinodai, 2005. 